\title{
Olfactory or auditory stimulation and their hedonic values differentially modulate visual working memory
}

\author{
ANA M. DONOSO, VÍCTOR FAUNDES, FELIPE FALCÓN, PAULINA ESPARZA \\ and PEDRO E. MALDONADO
}

CENI, and Program of Physiology and Biophysics, Faculty of Medicine, University of Chile, Santiago, Chile All authors contributed equally to this work.

\begin{abstract}
Working memory (WM) designates the retention of objects or events in conscious awareness when these are not present in the environment. Many studies have focused on the interference properties of distracter stimuli in working memory, but these studies have mainly examined the influence of the intensity of these stimuli. Little is known about the memory modulation of hedonic content of distracter stimuli as they also may affect WM performance or attentional tasks. In this paper, we have studied the performance of a visual WM task where subjects recollect from five to eight visually presented objects while they are simultaneously exposed to additional - albeit weak- auditory or olfactory distracter stimulus. We found that WM performance decreases as the number of items to remember increases, but this performance was unaltered by any of the distracter stimuli. However, when performance was correlated to the subject's perceived hedonic values, distracter stimuli classified as negative exhibit higher error rates than positive, neutral or control stimuli. We demonstrate that some hedonic content of otherwise neutral stimuli can strongly modulate memory processes.
\end{abstract}

Key terms: Humans, Odor Hedonic, Visual Memory, and Multimodal Memory.

\section{INTRODUCTION}

Working memory (WM) designates the retention of objects or events in conscious awareness when these are not present in the environment. It also refers to retention manipulation and its use in guiding behavior (Romanski, 2004). This cognitive ability participates in many essential behaviors, such as planned movements, learning and language comprehension (Baddeley, 1986; Jonides, 1995). According to the multiple components model (Baddeley, 1996), WM is composed of three systems, one of attention control (or central executive) and the other two independent are buffers for the storage of verbal and of visual-spatial information. The central executive system actively focuses attention on specific events and possesses a very limited capacity of retention (Miller, 1956). This system regulates the flow of information through the two other systems, which maintain the memory for its transitory utilization. Electrophysiological recordings have identified the prefrontal cortex as instrumental in WM tasks and as a neural substrate for the storage buffers of working memory (Bustos, Basoalto, Pinto-Hamuy, 2003; Goldman-Rakic, 1987). The prefrontal dorsolateral cortex (DLPFC), which exhibits interconnections with different cortical areas for behavioral control (Goldman-Rakic, 1987), also has connections with other sensitive and associative areas, like the inferotemporal and visual cortices (Suchan, Linnewerth, Koster, Daum, \& Schmid, 2006).

The anatomical relationship of DLPFC to primary sensory and association cortices suggests that these cortical areas not only 
might be used during WM but might also modulate WM in a multimodal manner. It has been proposed that the visual WM is codified as phonological information in the auditory cortex in order to be recalled with greater facility, but at the same time auditory stimuli, in turn, modulate WM in visual tasks (Valtonen, May, Mäkinen, \& Tintinen, 2003). An alternate model to multiple component theory of WM proposes that these functions arise through the coordinated recruitment, via attention, of brain systems that have evolved to accomplish sensory representation and action-related functions (Romanski, 2004). De Fockert et al. (De Fockert, Rees, Frith, \& Lavie, 2001) proposed that WM function controls selective visual attention in the brain. They suggested that WM capacity requires reducing the intrusion of irrelevant distracter stimuli to a minimum in order to maintain the most prominent stimuli activated. Some studies have directly addressed the interaction of different sensory modalities in WM. It has been demonstrated that performance decreases if a visual WM task is carried out while subjects are exposed to irrelevant auditory stimuli, even when subjects are explicitly instructed to ignore the auditory stimulus (Valtonen, May, Mäkinen, \& Tintinen, 2003). On the other hand, a previous study exposed subjects to a simultaneous WM and olfactory task, in order to study functional interference between both tasks (García, 1992). This study concluded that olfactory stimulation did not produce deterioration in the performance by the subjects memory tasks, not even as a distracter element. A recent study has shown that even environmental odorants can differentially modulate processes involved in visual attention, and that this influence depends on the characteristics of the odorants (Michael, Jacquot, Millot, \& Brand, 2005). Besides multimodal influences, hedonic content of distracter stimuli may also affect WM performance or attentional tasks. Emotional stimuli may influence DLPFC activity during WM but only when it is required by task conditions (Perlstein, Elbert, \& Stenger, 2002; Pollack, 1972). In order to clarify the relationship of distracter stimulus and the contribution to memory modulation by the hedonic values of these distracters, we studied the performance of a visual WM task when subjects are simultaneously exposed to distracter auditory or olfactory stimulation. The aim was to examine a possible correlation between changes in performance during a WM task with the reported hedonic values of the distracters, when they do not have sufficient intensity to impede normal performance.

\section{MATERIALS AND METHODS}

Subjects: All experiments on human subjects were conducted in accordance with the Declaration of Helsinki, and all procedures were carried out with the adequate understanding and written consent signed by the subjects. The experimental protocol and written consent form was approved by the Ethics Committee on Human research at the Faculty of Medicine, University of Chile. We recruited 80 college students (40 males, 40 females) who ranged from 17 to 30 years of age. The sample was entirely composed of students from medical-related careers and shared similar educational and intellectual abilities. All subjects reported normal audition and normal or corrected vision, with no reported respiratory illnesses.

Working memory task.

The subjects were instructed to visually recollect the images of a circular array of 5 to 8 object presented simultaneously in a computer screen. The object presented in each trial was randomly selected form a set of 12 possible related objects. We used four sets of objects, which were grouped in different categories (food, animals, clothing and household items). For each session, a single stimulus category was used and 24 different images were presented. Each session lasted approximately 10-15 minutes. The subjects signaled the onset of each trial by pressing a button. Then an image with objects appeared in a screen located in front of the subjects for 8 seconds. Then the image was turned off and the subject pressed the buttons in the pad of 
those objects he recollected from the images. The subject had up to 20 seconds to complete the task. No verbal or typing action was involved. Figure 1 depicts a scheme of the experimental paradigm.

Distracter stimulation. During each trial, the subject was seated in front of a computer monitor with his/her chin resting in a holder. A funnel connected to a computer-controlled olfactometer was located in front of the subject's nostrils, with a constant flow of pure air drawn from a compressed tank obtained by a commercial supplier (AGA, SA). The subjects also wore a pair of headphones connected to the control computer. During the 8-sec image presentation one of five possible conditions was presented: 1) olfactory stimulation with hexanal (dilution $0.5 \%$, Merck) or 2) olfactory stimulation with artificial honeydew odorant (dilution $11 \%)$, 3) auditory stimulation with instrumental music from http// recursos.cnice.mec.es, or 4) auditory stimulation with a "booing" recording, or 5) no stimulation, which served as a control mechanism. The "booing" recording depicted several people shouting in disapproval and was obtained from a web site (www.partnersinrhyme.com). Hexanal odorant and the booing recording were initially used as unpleasant stimulation and honeydew odorant and instrumental music as pleasant stimulation. This hedonic classification was arbitrarily established by preliminary exposures to several types of stimuli explored by the authors. The intensity of the auditory stimulation was set to $60 \mathrm{~dB}$ and the olfactory stimulation although at different concentration was rehearsed by the experimenters to be perceptible in a single sniff. In each session, eight control trials and four trials of each of the other conditions were presented. Each subject participated with a single session and was assigned at random one of the four stimulus categories.

Data acquisition and analysis. A control computer running custom-made software developed in LabWindows CVI (National Instruments) handled the entire experiment. An A/D card monitored the olfactometer and the response from the subjects. The responses were saved in a file; an automatic algorithm quantified the correct responses, and the total time used to

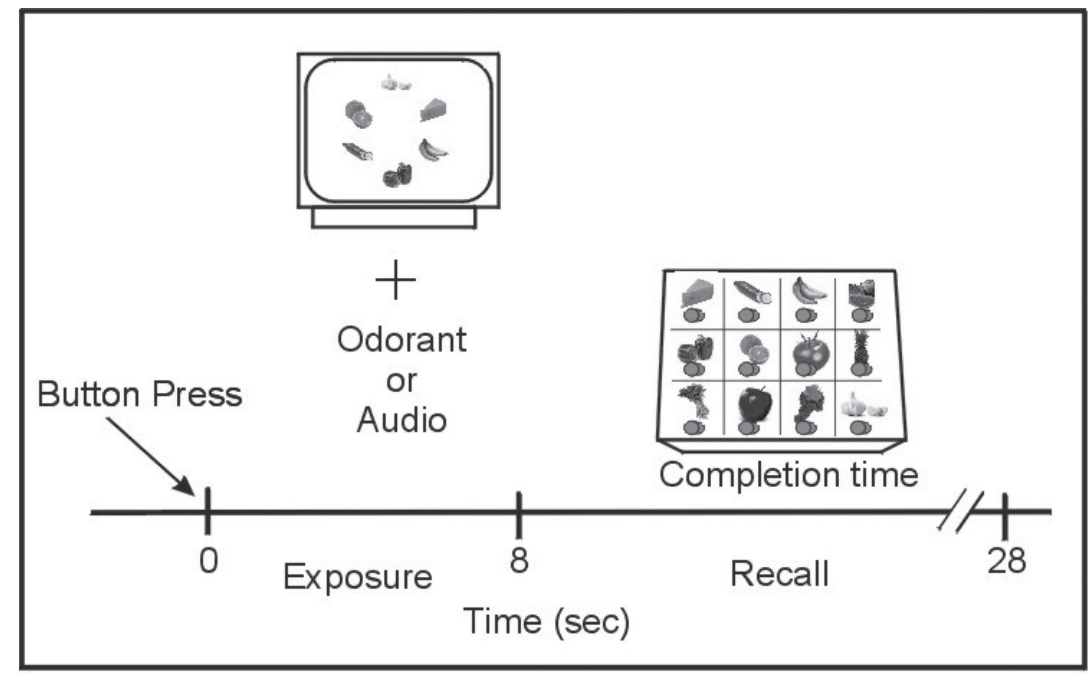

Figure 1: Scheme of the experimental working memory task. The subjects had to memorize 5-8 objects presented in a computer screen. When the image was turned off the subjects pressed the buttons in the pad of those recollected objects. The subjects had up to $20 \mathrm{sec}$ to complete the task. During each trial, an olfactometer could deliver an olfactory stimulation (hexanal or honeydew odorant). Alternatively, an auditory stimulation was delivered while the subjects were exposed to the objects. 
respond. A bell ring signaled the subject that the trial was completed or that the allowed time for response was over. Statistical analysis of the data was performed by using commercial software (StatMost 3.2).

Post-session survey. Once each experimental session was completed, subjects were asked to fill out a survey of his/her experience. Since the hedonic values of different stimuli could vary among individuals, we developed an hedonic scale based on three categorizes: a positive stimulus, which was described by the subjects as a pleasant one; a negative stimulus that fell under the category of unpleasant, and a neutral one when subjects were unable to classify it as any of the others categories. This survey was aimed to establish subjective values of hedonic content. Subjects were asked to rate each stimulus as neutral, positive or negative. This classification was then used to compare memory performance directly with the subject's experience.

\section{RESULTS}

We directed 80 sessions, one with each subject. First, we sorted the responses by the subject's gender and type of stimulus. The main measure of memory performance was based on the number of errors. Two types of errors were committed: commission errors where subject pressed buttons of objects not present during that stimulus exposure, and omission errors where they failed to press the number of buttons corresponding to the number of objects presented in that trial. We observed that nearly all errors were of the first type, so we classified all errors in a single group.

We initially examined if the memory task revealed the increased memory load as the number of objects presented in the games increased. This is revealed as an increased number of errors with larger number of objects that needed memorization. Since we present four different numbers of objects, we expressed the number of errors as a fraction of the total number of objects. A normality
Kolmogorov-Smirnov and Lilliefors test showed that these distributions were not normal $(\mathrm{P}<0.01)$. Thus, we employ nonparametric statistics to compare the fraction of errors for different data groups. Figure $2 \mathrm{~A}$ shows the statistics of the fraction of errors for all subjects for the control condition. As expected, the fraction of errors increases with memory load. We observed an incremental and significant difference between all stimulus sets ( $p$ $<0.01$ Kuskal-Wallis), when we compared each successive pairs of data, except between 7 and 8 objects. Along with the increase in the fraction of errors with the increased number of objects to memorize, we also observed a systematic and significant increase ( $p<0.01$ KuskalWallis) in the completion time. Figure 2Asummarizes these results. In this figure, we excluded from the distributions those few cases where times exceeded the maximum allowed to respond $(20 \mathrm{sec})$. When this data was separated by gender, we found no differences that would indicate a difference between the performance of males and females.

We examined the overall error incidence during the control versus the auditory and olfactory stimuli responses. We found that the number of errors in the memory task was lower during the control condition. However, we found no statistically significant difference for any of the stimulation condition when compared to the control condition. If we examine the fraction of errors for different numbers of objects, we find that as with the control, errors for olfactory and auditory stimuli systematically increase with memory load. (Figure 2B). Moreover, in our examination we found that this dependency was not statistically different between controls and distracter stimuli for any number of objects. This result demonstrates that the inclusion of distracter stimuli with these intensities interferes little with this WM task. This is confirmed by comparing the response errors during control to the four types of distracter stimulus, as shown in figure $3 \mathrm{~A}$, where the fraction of errors for distracter stimulus were always larger than control, but never reached significance $(\mathrm{P}>0.05)$. 
Finally, we examined the performance of the WM task in relation to the subject's perception of the hedonic value of the distracter stimuli. During the task, subjects were instructed to ignore all distracter stimuli. However, after the subject finished, we performed a survey where the subjects were asked to rate the four types of distracter in one of three categories: neutral, positive or negative. We then reviewed the performance of the subjects, classifying the responses based on the subject's assessment. The subject's hedonic characterization for all stimuli is shown in Table 1. As expected, hexanal and "booing" were mostly classified as negative, and, conversely, honeydew and music as positive. Interestingly, a large percentage of subjects were reportedly neutral to the distracters. Our results demonstrated that the intensity of the distracters was sufficiently low so as not to alter by themselves the performance of the task. Moreover, we found no significant differences in reaction times between these stimuli regardless of their hedonic value. When we computed task performance, we
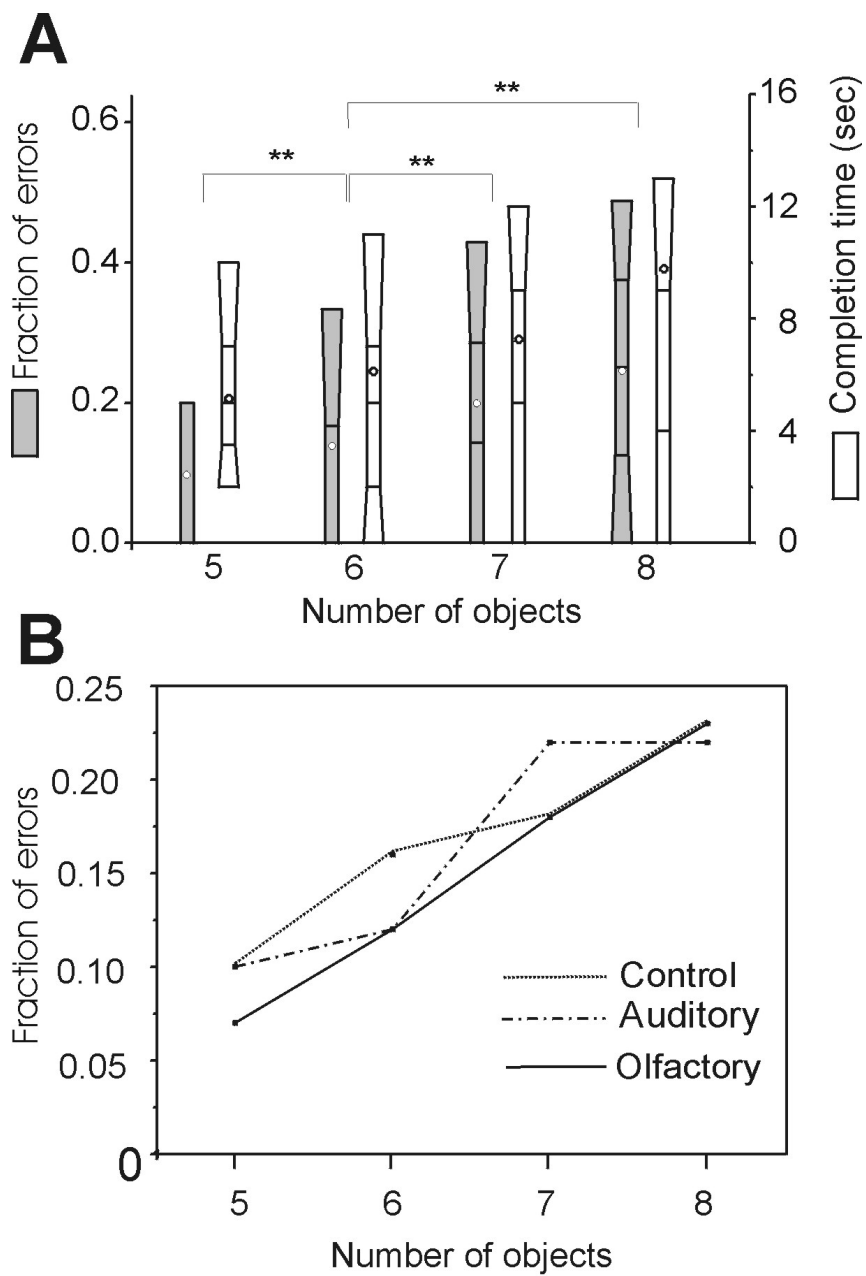

Figure 2: (A) Statistics of the fraction of errors in all subjects for the control condition. The fraction of errors increases with memory load (asterisks, $\mathrm{P}<0.01$ ). Along with the increase in the fraction of errors with the increased number of objects to memorize, we observed a systematic and significant increase $(\mathrm{p}<0.01)$ in completion time. The central point represents the median and the boxes represent the 50 and 75 percentiles respectively. (B) Increase relationship of errors with the number of objects is not altered by distracter stimuli. 
observed that stimuli classified as neutral or positive stimuli showed no significant differences in fraction of errors when compared to controls. However, stimuli that were rated as negative (whether auditory or olfactory) did significantly increase the fraction of errors in this WM task (Figure 3B). Positive or neutral stimuli exhibit similar fraction of errors as controls (Figure 3B). Interestingly, while the identity of the distracter stimulus seems to largely correlate with its hedonic classification, significance in the fraction of errors was not achieved by identity but only when pooling data from the subject's reported hedonic value. This result showed that the hedonic value and not the identity of the distracter was affecting the performance in this WM task.

\section{TABLE 1}

Subject categorization of hedonic value of distracter stimuli. A post-experiment survey of subjects recollects their categorization of distracter stimuli. Most stimuli were consistently, but not unanimously, found to be positive or negative.

\begin{tabular}{lccc}
\hline Stimulus & Neutral & Positive & Negative \\
\hline Hexanal & 30.4 & 8.7 & 60.9 \\
Honeydew & 21.6 & 63.5 & 14.9 \\
Booing & 43.7 & 1.3 & 55.0 \\
Music & 46.6 & 49.3 & 4.1 \\
\hline
\end{tabular}
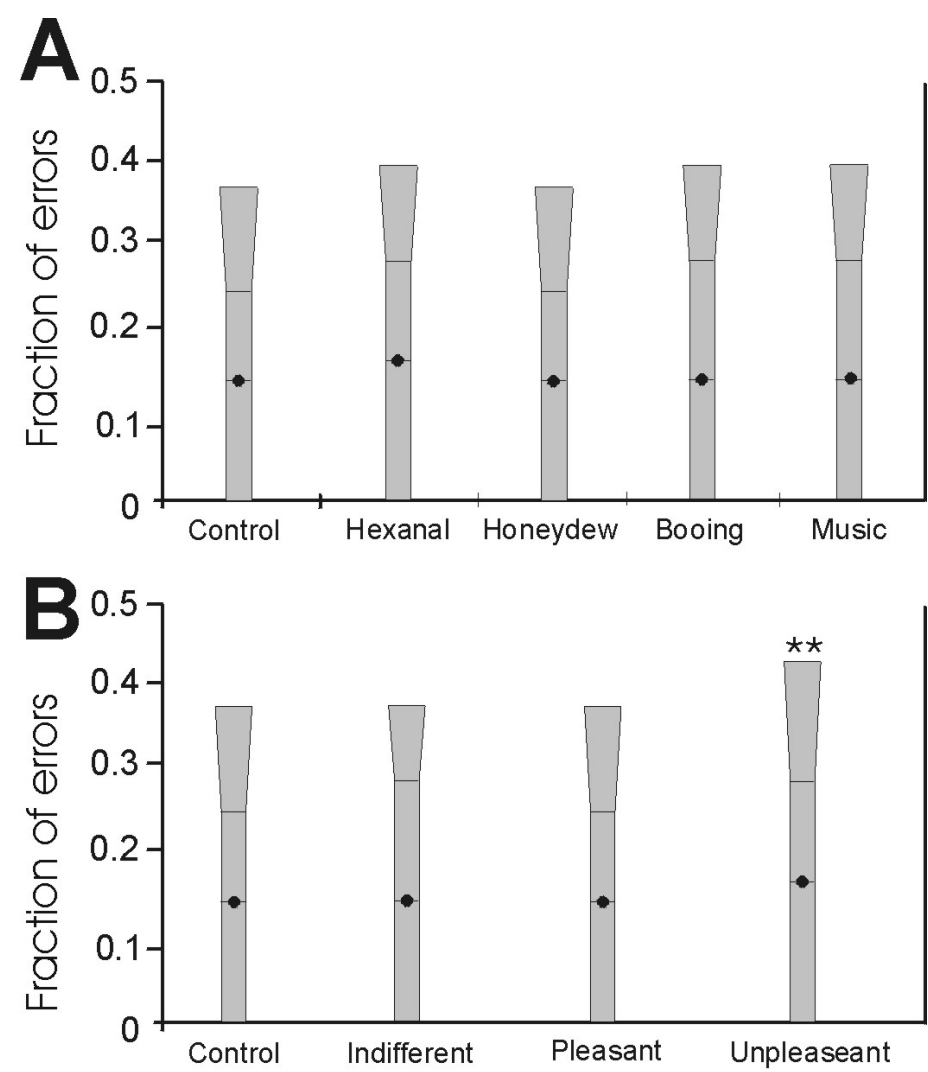

Figure 3: (A) Fraction of errors during control and the four types of distracter stimulus. Fractions of errors for distracter stimulus were not different from controls $(\mathrm{P}>0.05)$. (B) Hedonic value modulates WM error rates. Task performance for neutral or positive stimuli showed no significant differences in fraction of errors when compared to controls. Negative (whether auditory or olfactory) stimuli significantly increased the fraction of errors in this WM task. For all plots, the central points represent the median and the boxes represent the 50 and 75 percentiles. 


\section{DISCUSSION}

Our main objective was to analyze whether the integration of auditory and olfactory sensory modalities and the emotional component of stimuli are capable of modulating a visual WM task. WM is defined by its limited retention capacity, thus by enlarging the number of images to simultaneously memorize, we were increasing the difficulty of the task and consequently we observed a systematic decrease in performance. The subject's number of errors and response time increased as larger numbers of images were presented. No significant difference was observed in the number of errors when showing seven or eight images simultaneously. One likely explanation is that by requesting the memorization of this number of images the maximum retention capacity of the working memory is achieved (Miller, 1956). Other studies have shown that subjects can memorize up to $7 \pm$ 2 items simultaneously (Postle, 2006), but recent evidence has reexamined this issue proposing a lower limit. (Cowan, 2001; Luck, \& Vogel, 2007). It is important to emphasize that in these studies, the exposition time of the stimuli was more than the necessary for his cortical integration. Therefore the performance of the subjects may include the full effect of hedonic values on WM. Our experiment considered a prolonged stimulus exposition time and yet our results show that when we compare the performance of the subjects under distinct distracting stimuli, we found there were no significant differences in WM for the different distracter categories.

In our study, it was important that the distracter stimuli did not interfere with the WM task primarily by their intensity. Numerous studies show that distracter stimuli, if intense enough, may interfere with performance (Baddeley, \& Della Sala, 1998; Jonides, \& Nee, 2006; Michael, Jacquot, \& Millot, 2003; Postle, 2006). However, when the distracter has lower intensities, WM itself can modulate attention and focus towards what needs to be memorized, despite the presence of these weak distracter (Berti, \& Schröger, 2003;
Hall, \& Blasko, 2005). In these studies, significant interferences were observed in attention towards novel stimulus or significant for subjects who exhibited reduced performance in a WM task. In our experiments we did not find any significant difference in reaction time or number of errors for all distracters. Clearly, none were a novel stimuli and we found no significant inter subject difference, thus we conclude that neither the identity, nor the intensity or novelty of the distracter stimulus was enough to cause interference in this task.

Though quantitative hedonic scales have been widely used in other studies of working memory, our main goal was to examine whether the presentation of an additional stimulus with a hedonic component had an effect on a working memory task. Therefore in our study, analyzing the quality of the stimulus was more useful to this purpose than using the magnitude of the influence.

When analyzing the effect hedonic value in our WM task, we observed that distracter stimuli described by the subjects as "negative" increased the number of errors. At the same time, no significant difference in the response time was observed. This demonstrates an integration mode between the emotional activation of the brain and WM. Although studies have shown differences in WM task involving images with emotional contents (Maljkovic, \& Martin, 2005; Perlstein, Elbert, \& Stenger, 2002; Pollack, 1972), these studies demonstrated that the capacity of memorizing image was increased when the stimulus was described by the subjects as negative, suggesting that attention resources to these stimuli increased. This could explain in part, that when our subjects described distracter stimulus as negative, the performance in the visual WM task decreased. Aside from this work, no other study has investigated how hedonic or emotional components of distracter stimulus sensorial modalities can alter attention and modulate visual WM.

One previous study described a direct modulation of attention arising from the olfactory pathway (Michael, Jacquot, Millot, \& Brand, 2003). They observed 
deterioration in the performance of WM tasks mainly when the olfactory stimuli was an irritant by activating the trigeminal pathway. We cannot rule out that subjects that classified olfactory stimulation as "negative" were under trigeminal activation. However, this cannot be the only explanation for the performance differences for hedonic value, since auditory distracter classified as negative also exhibited an increment if the fraction of errors.

This study sustains the hypothesis that the activity of the DLPFC is affected for the emotional qualities of the stimulus. In this regard the amygdala would play a fundamental role as a cerebral structure maintaining string connections with other regions of the brain (frontal and temporal lobes), which enables evaluation of emotional or hedonic meaning of events. Thus, the amygdala may be capable of facilitating memory operations and of acting as a mediator in the emotional learning (Goldman-Rakic, 1996). "According to this, the existence of areas that codify this emotional information and relate it to the WM system (Compton. 2003), appears necessary. Through them, those stimuli that have a greater emotional meaning will have a priority set against others in the access to selective attention All this implies bottom-up entrance to the amygdala as well as top-down influences of the frontal lobe regions implied in representations that set and maintain goaloriented behavior in WM. In this way, the prefrontal region modulates the responses that the amygdala provides, comparing potential risks and benefits of possible actions. Despite all this, it's not possible to completely reject the theory that states that our findings were a result of misdirected attention towards the distracting stimuli.

Our main objective was to analyze if the integration of the auditory and olfactory sensory modalities and its hedonic components, are capable of modulating a working memory task. Although it is well known that many phenomena such as the attention itself or arousal can alter the stimuli perception, our study tried to examine its final consequences and not the mechanism with which they are integrated.
Yet, besides the distracter capacity of a stimulus is given by intensity, we conjecture that its emotional or hedonic content may also affect memory tasks. We demonstrate that while the low intensity of distracters does not affect this WM task, the hedonic value does, implying that these characteristics can strongly modulate memory processes.

\section{ACKNOWLEDGEMENTS}

This study was supported by Iniciativa Científica Milenio ICM P04-068F.

Dedicated to Dr. Teresa Pinto-Hamuy.

\section{REFERENCES}

BADDELEY AD (1986) Working Memory, Oxford University Press, London.

BADDELEY AD (1966) The fractionation of working memory, Proceedings of the National Academy of Science, 93, 13468-13472.

BADDELEY AD, DELLA SALAS (1998) Working memory and executive control. In: A.C. Roberts, T. W. Robbins, L. Weiskrantz (Eds.), The prefrontal cortex: Executive and cognitive functions, Oxford University Press, New York, pp. 9-21.

BERTI S, SCHRÖGER E (2003) Working memory controls involuntary attention switching: evidence from an auditory distraction paradigm. European Journal of Neuroscience, 17, 1119-1122.

BUSTOS G, BASOALTO E, PINTO-HAMUY T (2003) Spatial memory in long evans and rattus norvegicus rats. Biological Research, 36, 193-199.

COMPTON RJ (2003) The interface between emotion and attention: A review of evidence from psychology and neuroscience. Behavioral and Cognitive Neuroscience Reviews, 2, 115-129.

COWAN N (2001) The magical number 4 in short-term memory: a reconsideration of mental storage capacity. Behavioral Brain Science. 24, 87-114.

DE FOCKERT JW, REES G, FRITH CD, LAVIE N (2001) The role of working memory in visual selective attention. Science, 291, 1803-1806.

GARCÍA LM (1992) Memoria y Olfato: Interferencias funcionales, Universidad Complutense de Madrid.

GOLDMAN-RAKIC PS (1987) Circuitry of the prefrontal cortex and the regulation of behavior by representational memory. In: V. B. Mountcastle, F. Plum, S. R. Geiger (Eds.), Handbook of Physiology, American Physiological Society, Bethesda, pp. 373417.

GOLDMAN-RAKIC PS (1996) The prefrontal landscape: implications of functional architecture for understanding human mentation and the central executive. Philosophical Transactions Royal Society London B Biological Sciences, 351, 1445-1453.

HALL M.D, BLASKO DG (2005) Attentional interference in judgments of musical timbre: individual differences in working memory. Journal General Psychology, 132, 94-112. 
JONIDES J (1995) Working memory and thinking. In: E. E. Smith, D. N. Osherson (Eds.), An Invitation to Cognitive Science, Vol. 3, MIT Press, Cambridge, pp. 215-265.

JONIDES J, NEE DE (2006) Brain mechanisms of proactive interference in working memory. Neuroscience, 139,181-193.

LUCK SJ, VOGEL EK (1997) The capacity of visual working memory for features and conjunctions. Nature, 390, 279-281.

MALJKOVIC V, MARTINI P (2005) Short-Term memory scenes with affective contents. Journal of Vision, 5, 215-229.

MICHAEL GA, JACQUOT L, MILLOT JL, BRAND G (2003) Ambient odors modulate visual attention capture. Neuroscience Letters, 352, 221-225.

MICHAEL GA, JACQUOT L, MILLOT JL, BRAND G (2005) Ambient odors influence the amplitude and time course of visual distraction. Behavioral Neuroscience, $119,708-715$.

MILLER GA (1956) The magical number seven, plus or minus two: some limits on our capacity for processing information. Psychology Review, 63, 81-97.

PERLSTEIN WM, ELBERT T, STENGER VA (2002) Dissociation in human prefrontal cortex of affective influences on working memory-related activity. Proceedings of the National Academy of Science, U S A, 99, 1736-1741.

POLLACK I (1972) Memory for auditory waveform. Journal Acoustical Society America, 52, 1209-1215.

POSTLE BR (2006) Working memory as an emergent property of the mind and brain. Neuroscience, 139, 2338.

ROMANSKI LM (2004) Domain specificity in the primate prefrontal cortex. Cognitive and Affective Behavioral Neuroscience, . 4, 421-429.

SUCHAN B, LINNEWERTH B, KOSTER O, DAUM I, SCHMID G (2006) Cross-modal processing in auditory and visual working memory. Neuroimage, 29, 853-858.

VALTONEN J, MAY P, MÄKINEN V, TIITINEN H (2003) Visual short-term memory load affects sensory processing of irrelevant sounds in human auditory cortex. Brain Research: Cognitive Brain Research, 17, $358-367$. 